\title{
M-IDEALS OF COMPACT OPERATORS IN CLASSICAL BANACH SPACES
}

\author{
ÅSVALD LIMA
}

\section{Abstract.}

Let $K(X, X)$ be the space of compact operators on an infinite dimensional Banach space $X$. It is known that $K(X, X)$ is an $\mathrm{M}$-ideal (in the space of all bounded operators on $X$ ) when $X=L_{2}(\mu)$ for some measure $\mu$. We prove that:

1) If $X=L_{1}(\mu)$, then $K(X, X)$ is not an M-ideal.

2) If $X^{*}=L_{1}(\mu)$ then $K(X, X)$ is an M-ideal iff $X=c_{0}(\Gamma)$.

3) If $1<p<\infty, p \neq 2$, and $X=L_{p}(\mu)$, then $K(X, X)$ is an M-ideal iff $\mu$ is purely atomic.

\section{Introduction.}

The object of this paper is to investigate when $K(X, Y)$, the space of compact operators from $X$ to $Y$, is an $M$-ideal in $L(X, Y)$, the space of all bounded operators from $X$ to $Y$.

When Alfsen and Effros [1] introduced the notion of an M-ideal, they knew that in the self-adjoint part of a $\mathrm{C}^{*}$-algebra, the $\mathrm{M}$-ideals coincide with the selfadjoint parts of the closed two-sided ideals. Later Smith and Ward [12] proved that the $\mathrm{M}$-ideals in a $\mathrm{C}^{*}$-algebra are exactly the closed two-sided ideals. In particular, the compact operators on a Hilbert space is an M-ideal in the space of all bounded operators.

Hennefeld [2] and Saatkamp [11] have proved that $K\left(l_{p}, l_{q}\right)$ are M-ideals when $1<p \leqq q<\infty$ and several authors have observed that $K\left(X, c_{0}\right)$ is an $\mathrm{M}$ ideal for all Banach spaces $X$ [6] [11] [12]. Note that if $1 \leqq q<p<\infty$, then $K\left(l_{p}, l_{q}\right)=L\left(l_{p}, l_{q}\right)[8]$.

It is known that $K\left(l_{1}, l_{1}\right)$ and $K\left(l_{\infty}, l_{\infty}\right)$ are not $\mathrm{M}$-ideals [12] and also in some other cases involving $L_{p}$-spaces and preduals of $L_{1}$-spaces $K(X, Y)$ is not an M-ideal [9] [11].

The paper consists of two parts. In the first part, we show that the $X=c_{0}(\Gamma)$ are the only Lindenstrauss spaces such that $K(X, X)$ is an M-ideal. In the second part, we prove some theorems saying that if $X$ and $Y$ have some properties (this is specified later), then $K(X, Y)$ is not an M-ideal. 
When we say that $K(X, Y)$ is an $\mathrm{M}$-ideal, we mean that $K(X, Y)$ is an $\mathrm{M}$ ideal in $L(X, Y)$. A Banach space $X$ is called a Lindenstrauss-space if its dual $X^{*}$ is isometric to an $L_{1}(\mu)$-space. The unit ball in $X$ is denoted $X_{1}$ and the closed ball in $X$ with center $x$ and radius $r$ is denoted $B(x, r)$.

A closed subspace $J$ of a Banach space $A$ is called an $L_{p}$-summand $(l \leqq p$ $<\infty$ ) if there exists a projection $P$ in $A$ such that $P(A)=J$ and for all $x \in A$ we have

$$
\|x\|^{p}=\|P x\|^{p}+\|x-P x\|^{p} .
$$

A closed subspace $J$ of $A$ is called an M-summand if $J$ is the range of a projection $P$ in $A$ such for all $x \in A$ we have

$$
\|x\|=\max (\|P x\|,\|x-P x\|) .
$$

A closed subspace $J$ of $A$ is called an M-ideal if its annihilator $J^{\circ}$ in $A^{*}$ is an $L_{1}$-summand. Alfsen and Effros [1] characterized M-ideals by intersection properties of balls. In [5] we showed that a closed subspace $J$ of $A$ is an Mideal iff for all $x \in A_{1}$, for all $y_{1}, y_{2}, y_{3} \in J_{1}$ and for all $\varepsilon>0$, there exists

$$
y \in J \cap \bigcap_{i=1}^{3} B\left(x+y_{i}, 1+\varepsilon\right) .
$$

If $\left({ }^{*}\right)$ holds when $y_{1}=y_{2}=-y_{3}$, then we say that $J$ is a semi M-ideal. We have that $J$ is a semi M-ideal iff for all $x \in A^{*}$, there exists a unique $y \in J^{\circ}$ such that $\|x-y\|=d\left(x, J^{\circ}\right)$ and moreover this unique $y$ satisfies $\|x\|=\|y\|+\|x-y\|$. [5; Theorem 6.15 and Theorem 5.6.]

The set of extreme points of a convex set $C$ is denoted $\partial_{e} C$.

We say that a point $e \in A$ is an order unit for $A$ if $\|e\|=1$ and

$$
\max (\|x+e\|,\|x-e\|)=\|x\|+1
$$

for all $x \in A$. This definition is equivalent to the usual definition of order unit [7; Theorem 4.7]. (See also [6]).

A maximal proper face $F$ of $A_{1}$ is called a base if $A_{1}=\operatorname{co}(F \cup-F)$. If $F$ is a base for $A$, then the functional on $A$ which is 1 on $F$ is an order unit for $A^{*}$. If $e$ is an order unit in $A^{*}$ and

$$
F=\{x \in A:\|x\|=1=e(x)\},
$$

then $A_{1}=\overline{c o}(F \cup-F)[6]$. (The bar means closure and co means convex hull.)

We consider only the real case, but most of the results are easily extended to the complex case. 


\section{Characterization of $c_{0}(\Gamma)$.}

Note that if $X$ or $X^{*}$ is an $L_{1}(\mu)$-space, then $e$ is an order unit for $X^{*}$ for all $e \in \partial_{e} X_{1}^{*}$. We will use this property in the first lemma.

Lemma 1. Suppose $e$ is an order unit for $X^{*}$ for all $e \in \partial_{e} X_{1}^{*}$. If $K(X, X)$ is a semi $\mathrm{M}$-ideal in $L(X, X)$, then $X$ is isometric to a subspace of $c_{0}(\Gamma)$ for some set $\Gamma$. If $X$ is separable, then we can take $\Gamma$ to be countable.

Proof. Note that if $f, g \in \hat{o}_{e} X_{1}^{*}$ with $f \neq g$, then $\|f-g\|=2$ [6; Theorem 2.2]. Choose $e \in \partial_{e} X_{1}^{*}$ and $\varepsilon>0$ and let $x_{1} \in X$ with $\left\|x_{1}\right\|=1$. Define

$$
N=\left\{f \in \partial_{e} X_{1}^{*}:\left|f\left(x_{1}\right)\right| \geqq 2 \varepsilon\right\} .
$$

We want to show that $N$ is finite.

Define $S \in K(X, X)$ by $S(x)=e(x) x_{1}$. Then $\|S\|=1$. Since $K(X, X)$ is a semi $M$-ideal there exists an operator

$$
U \in K(X, X) \cap B(I-S, 1+\varepsilon) \cap B(I+S, 1+\varepsilon) .
$$

Thus

$$
\|S+(I-U)\| \leqq 1+\varepsilon,\|S-(I-U)\| \leqq 1+\varepsilon .
$$

If $f \in N$, then $S^{*} f=f\left(x_{1}\right)$. Hence

$$
\begin{aligned}
1+\varepsilon & \geqq \max \left(\left\|f\left(x_{1}\right) e+\left(f-U^{*} f\right)\right\|,\left\|f\left(x_{1}\right) e-\left(f-U^{*} f\right)\right\|\right) \\
& =\left|f\left(x_{1}\right)\right|+\left\|f-U^{*} f\right\| \\
& \geqq 2 \varepsilon+\left\|f-U^{*} f\right\|
\end{aligned}
$$

and $1-\varepsilon \geqq\left\|f-U^{*} f\right\|$.

But then we get for $f, g \in N$ with $f \neq g$,

$$
\left\|U^{*} f-U^{*} g\right\| \geqq\|f-g\|-\left\|f-U^{*} f\right\|-\left\|g-U^{*} g\right\| \geqq 2 \varepsilon .
$$

Since $U^{*}$ is compact, we get that $N$ is finite, Now we can take $\Gamma=\partial_{e} X_{1}^{*}$. By considering $x_{1}$ as a function on $\partial_{e} X_{1}^{*}$, we get $x_{1} \in c_{0}(\Gamma)$. If $X$ is separable, we can take as $\Gamma$ a countable $\omega^{*}$-dense subset of $\hat{\partial}_{e} X_{1}^{*}$.

Theorem 2. Suppose $X$ is a Lindenstrauss space. Then the following statements are equivalent.

1) $X$ is isometric to $c_{0}(\Gamma)$ for some set $\Gamma$.

2) $K(Y, X)$ is an $M$-ideal in $L(Y, X)$ for all Banach spaces $Y$.

3) $K(X, X)$ is an M-ideal in $L(X, X)$.

4) $K(X, X)$ is a semi M-ideal in $L(X, X)$. 
Proof. 1) $\Rightarrow$ 2) is proved in [6], [11] and [12].

2) $\Rightarrow 3$ ) $\Rightarrow 4$ ) is trivial.

4) $\Rightarrow 1$ ). Let $F$ be a proper maximal face of $X_{1}^{*}$ and let $\Gamma=\partial_{e} X_{1}^{*} \cap F$. By the lemma above, we get $X \subseteq c_{0}(\Gamma)$ by the natural map. We have $X_{1}^{*}=\operatorname{co}(F \cup-F)$, so $\partial_{e} X_{1}^{*}=(\Gamma \cup-\Gamma)[5]$. Since $X$ necessarily is polyhedral, we get $X^{*}=l_{1}(\Gamma)$ $=c_{0}(\Gamma)^{*}[4][10]$. It follows from the Hahn-Banach theorem that $X=c_{0}(\Gamma)$.

The same method of proof as used to prove the theorem above can be used to prove the following result.

THeOrem 3. Suppose $X$ is a Lindenstrauss space and assume $X$ is canonically imbedded into $X^{* *}$. Then the following statements are equivalent.

1) $X$ is isometric to $c_{0}(\Gamma)$ for some set $\Gamma$.

2) $X$ is an M-ideal in $X^{* *}$.

3) $X$ is a semi $\mathrm{M}$-ideal in $X^{* *}$.

Proof. 1) $\Rightarrow 2$ ). We have that for each finite set $A \subseteq \Gamma$,

$$
l_{\infty}^{A}=\left\{(x(\gamma)) \in l_{\infty}(\Gamma): x(\gamma)=0 \text { if } \gamma \notin A\right\}
$$

is an M-summand in $X^{* *}=l_{\infty}(\Gamma)$. Hence

$$
c_{0}(\Gamma)=\overline{\bigcup l_{\infty}^{A}}
$$

(the union taken over all finite subsets $A$ of $\Gamma$ ) is an M-ideal in $X^{* *}$ by [5; Proposition 6.20].

2) $\Rightarrow 3$ ) is trivial.

3) $\Rightarrow 1$ ). Choose $x \in X$ with $\|x\|=1$ and let $\varepsilon>0$. First we want to show that

$$
N=\left\{e \in \partial_{e} X_{1}^{*}:|e(x)| \geqq \varepsilon\right\}
$$

is finite. Choose $y \in \partial_{e} X_{1}^{* *}$. Then $|y(e)|=1$ for all $e \in \partial_{e} X_{1}^{*}$ [6; Theorem 2.2]. Use the balls $B(y+x, 1)$ and $B(y-x, 1)$ and proceed as in Lemma 1 to show that $N$ is finite. Then argue as in the proof of 4$) \Rightarrow 1$ ) in the proof of Theorem 2 , and it follows that $X$ is isometric to a $c_{0}(\Gamma)$ space.

Although it is well known that $K\left(l_{p}, l_{q}\right)$ is an M-ideal when $1<p \leqq q<\infty$, we would like to give a simple proof of this using the characterization $\left({ }^{*}\right)$.

TheOREM 4. $K\left(l_{p}, l_{q}\right), K\left(l_{p}, c_{0}\right)$ and $K\left(c_{0}, c_{0}\right)$ are M-ideals when $1<p \leqq q<\infty$.

Proof. We write out the details only in the case $K\left(l_{p}, l_{q}\right)$ with $1<p \leqq q<\infty$. Let $S_{1}, S_{2}, S_{3} \in K\left(l_{p}, l_{q}\right)$ with $\left\|S_{i}\right\| \leqq 1$ and let $T \in L\left(l_{p}, l_{q}\right)$ with $\|T\| \leqq 1$. Since we 
have an $\varepsilon>0$ at our disposal in the formula $\left({ }^{*}\right)$, we may suppose $S_{i}=Q_{m} S_{i} P_{n}$ for $i=1,2,3$ and some $m$ and $n$ where $Q_{m}$ and $P_{n}$ are the projections

$$
P_{n}\left(\left(x_{k}\right)\right)=Q_{n}\left(\left(x_{k}\right)\right)=\left(x_{1}, \ldots, x_{n}, 0, \ldots\right) .
$$

Let $U=Q_{m} T+T P_{n}-Q_{m} T P_{n} \in K\left(l_{p}, l_{q}\right)$. Then $T-U=\left(I-Q_{m}\right) T\left(I-P_{n}\right)$. Let $x \in l_{p}$ with $\|x\|=1$, and let $y=P_{n} x$ and $z=\left(I-P_{n}\right) x$. Then

$$
1=\|x\|^{p}=\|y\|^{p}+\|z\|^{p}
$$

and

$$
\begin{aligned}
\|y\| & =\left\|P_{n} x\right\| \geqq\left\|Q_{m} S_{i} P_{n} x\right\| \quad(i=1,2,3) \\
\|z\| & =\left\|\left(I-P_{n}\right) x\right\| \geqq\left\|\left(I-Q_{m}\right) T\left(I-P_{n}\right) x\right\| .
\end{aligned}
$$

Hence, since $S_{i}=Q_{m} S_{i} P_{n}$ for $i=1,2,3$,

$$
\begin{aligned}
1 & =\|y\|^{p}+\|z\|^{p} \\
& \geqq\|y\|^{q}+\|z\|^{q} \\
& \geqq\left\|Q_{m} S_{i} P_{n} x\right\|^{q}+\left\|\left(I-Q_{m}\right) T\left(I-P_{n}\right) x\right\|^{q} \\
& =\left\|Q_{m} S_{i} P_{n} x+\left(I-Q_{m}\right) T\left(I-P_{n}\right) x\right\|^{q} \\
& =\left\|S_{i} x+(T-U) x\right\|^{q} .
\end{aligned}
$$

This shows that

$$
U \in \bigcap_{i=1}^{3} B\left(T+S_{i}, 1\right)
$$

\section{Conditions which ensure that $K(X, Y)$ is not an M-ideal.}

The following theorem is an easy consequence of $\left({ }^{*}\right)$ and of [7; Theorem 6.1] and [13]. (We consider here only infinite dimensional spaces.)

Theorem 5. Let $Y$ be a Banach space. $K\left(l_{\infty}(\Gamma), Y^{*}\right)$ is an $\mathrm{M}$-ideal for all sets $\Gamma$ if and only if $K\left(X, Y^{*}\right)$ is an M-ideal for all Lindenstrauss spaces $X$. If $K\left(X, Y^{*}\right)$ is an $\mathrm{M}$-ideal for some infinite dimensional Lindenstrauss space $X$, then $K\left(c_{0}, Y^{*}\right)$ is an M-ideal.

The theorem remains true if we read semi $M$-ideals instead of $M$-ideals. Since $K\left(c_{0}, l_{\infty}\right)$ is not a semi M-ideal [11], we get that $K\left(X, l_{\infty}\right)$ is not a semi Mideal for any infinite dimensional Lindenstrauss space $X$.

It also follows from (*) that if $X$ and $Y$ are 1-complemented in $M$ and $N$ and $K(M, N)$ is an (semi) $M$-ideal, then $K(X, Y)$ is an (semi) $M$-ideal. 
Since $K\left(l_{1}, l_{p}\right)(1 \leqq p<\infty)$ and $K\left(l_{p}, l_{\infty}\right)(1<p<\infty)$ are not semi M-ideals [11], we get that $K\left(L_{1}(\mu), L_{p}(v)\right)(1 \leqq p<\infty)$ and $K\left(L_{p}(v), l_{\infty}\right)(1<p<\infty)$ are not semi $M$-ideals in the infinite dimensional cases. [3; Theorem 3].

As we will show now these results are special cases of more general results. Note that all maximal proper faces of the unit balls of $L_{1}(\mu)$-spaces and Lindenstrauss spaces are bases [5; Corollary 3.6]. These spaces also have the property that every extreme point in the dual unit balls is an order unit for the dual space.

THEOREM 6. Suppose $X^{*}$ is an order unit space with order unit $f$ and suppose $Y$ is an order unit space with order unit e. If $K(X, Y)$ is a semi $\mathrm{M}$-ideal in $L(X, Y)$, then $K(X, Y)=L(X, Y)$.

Proof. Let $F=\{x \in X:\|x\|=1=f(x)\}$. Then $\overline{c o}(F \cup-F)=X_{1}$ [6]. Hence, the compact operator $S$ defined by $S(x)=f(x) e$, has norm 1. Suppose $T \in L(X, Y)$ with $\|T\|=1$ and let $\varepsilon>0$. Then there exists

$$
U \in K(X, Y) \cap B(T+S, 1+\varepsilon) \cap B(T-S, 1+\varepsilon) .
$$

We get

$$
\max \|S \pm(T-U)\| \leqq 1+\varepsilon .
$$

Let

$$
G=\left\{y^{*} \in Y^{*}:\left\|y^{*}\right\|=1=y^{*}(e)\right\} .
$$

Then $Y_{1}^{*}=\operatorname{co}(G \cup-G)$. If $y^{*} \in \partial_{e} G$, then $S^{*} y^{*}=y^{*}(e) f=f$. Hence we get for $y^{*} \in \partial_{e} G$

$$
\begin{aligned}
1+\varepsilon & \geqq \max _{ \pm}\left\|f \pm\left(T^{*} y^{*}-U^{*} y^{*}\right)\right\| \\
& =1+\left\|\left(T^{*}-U^{*}\right) y^{*}\right\|
\end{aligned}
$$

Thus

$$
\|T-U\| \leqq \varepsilon
$$

Since $U \in K(X, Y)$ and $\varepsilon>0$ is arbitrary, we get $T \in K(X, Y)$.

THEOREM 7. Suppose $X^{*}$ is an order unit space with order unit $f$. Let $Y$ be a Banach space that contains a proper $L_{p}$-summand for some $1 \leqq p<\infty$. If $K(X, Y)$ is a semi M-ideal in $L(X, Y)$, then $K(X, Y)=L(X, Y)$.

Proof. Write $Y=E \oplus_{p} F$. For simplicity, assume $p=1$. Let $T \in L(X, Y)$ with 
$\|T\|=1$, and let $P$ be the $L_{p}$-projection in $Y$ with range $E$. Choose $x_{1} \in E$ with $\left\|x_{1}\right\|=1$ and let $\varepsilon>0$. Define $S \in K(X, Y)$ by $S(x)=f(x) x_{1}$. Then there exists

$$
U \in K(X, Y) \cap B(T+S, 1+\varepsilon) \cap B(T-S, 1+\varepsilon) .
$$

Hence

$$
\max \|S \pm(T-U)\| \leqq 1+\varepsilon .
$$

For $x \in X$ with $\|x\| \leqq 1$, we get

$$
\begin{aligned}
1+\varepsilon & \geqq\left\|f(x) x_{1}-(T-U) x\right\| \\
& =\left\|f(x) x_{1}-P(T-U) x-(I-P)(T-U) x\right\| \\
& =\left\|f(x) x_{1}-P(T-U) x+(I-P)(T-U) x\right\|
\end{aligned}
$$

and we also have

$$
1+\varepsilon \geqq\left\|f(x) x_{1}+P(T-U)+(I-P)(T-U) x\right\| .
$$

Hence we get when $x \in H=\{x \in X:\|x\|=1=f(x)\}$

$$
\begin{aligned}
2(1+\varepsilon) & \geqq\left\|f(x) x_{1}-P(T-U) x+(I-P)(T-U) x\right\| \\
& +\left\|f(x) x_{1}+P(T-U) x+(I-P)(T-U) x\right\| \\
& \geqq 2\left\|f(x) x_{1}+(I-P)(T-U) x\right\| \\
& =2\left\|x_{1}\right\|+2\|(I-P)(T-U) x\| .
\end{aligned}
$$

This together with $X_{1}=\overline{c o}(H \cup-H)$ yields

$$
\varepsilon \geqq\|(I-P)(T-U)\|=\|(I-P) T-(I-P) U\| .
$$

Thus $(I-P) T \in K(X, Y)$. Similarly, we get $P T \in K(X, Y)$ by choosing $x_{1} \in F$, so $T \in K(X, Y)$.

A proof similar to the proof of Theorem 7 shows that we also have the following result.

Theorem 8. Assume $X$ contains a proper $M$-ideal or a proper $L_{p}$-summand for some $1<p<\infty$, and assume $Y$ is an order unit space. If $K(X, Y)$ is a semi $\mathrm{M}$-ideal in $L(X, Y)$, then $K(X, Y)=L(X, Y)$.

A bounded subset $A$ of a Banach space $Y$ is said to be dentable if for all $\varepsilon>0$, there exists $t>0$ and $f \in Y^{*}$ with $\|f\|=1$ such that the slice

$$
S(f, t)=\left\{x \in A: f(x)>\sup _{a \in A} f(a)-t\right\}
$$


has diameter less than $\varepsilon$. In reflexive spaces and separable dual spaces, all bounded sets are dentable. [14].

THEOREM 9. Assume $X^{*}$ has an order unit $e$ and assume $Y_{1}$ is dentable. If $K(X, Y)$ is a semi M-ideal, then $K(X, Y)=L(X, Y)$.

Proof. Assume $K(X, Y)$ is a semi $\mathrm{M}$-ideal and let $T \in L(X, Y)$ with $\|\mathrm{T}\|=1$.

Assume for contradiction that $d(T, K(X, Y))>\varepsilon$ and $\varepsilon>0$. Let $S(f, t)$ be a slice of $Y_{1}$ with diam $S(f, t)<\varepsilon$. By the Bishop-Phelps theorem, we may assume $\|f\|=1=f(y)$ for some $y \in S(f, t) \subseteq Y_{1}[14]$. Define $S \in K(X, Y)$ by $S(x)=e(x) y$. Choose $0<\delta<1$ such that $(1-\delta)(1+\delta)^{-1}>1-t$. Since $K(X, Y)$ is a semi Mideal, there exists $U \in K(X, Y)$ such that for both \pm :

$$
\|S \pm(T-U)\| \leqq 1+\delta .
$$

Since $\|T-U\|>\varepsilon$, there exists $x \in X_{1}$ with $e(x)=1$ such that $\|(T-U) x\|>\varepsilon$. Then $S(x)=y$. Let $z=(T-U) x$. Then

$$
\max \|y \pm z\| \leqq 1+\hat{\partial}
$$

such that

$$
1+\delta>f(y \pm z)=1 \pm f(z)
$$

and

$$
|f(z)| \leqq \delta
$$

But then

$$
f\left(\frac{y \pm z}{1+\delta}\right) \geqq \frac{1-\delta}{1+\delta}>1-t
$$

so $(y \pm z)(1+\delta)^{-1} \in S(f, t)$. diam $S(f, t)<\varepsilon$ implies that

$$
2 \varepsilon<2\|z\|=\|(y+z)-(y-z)\|<\varepsilon(1+\delta)
$$

such that $\delta>1$. This is a contradiction. Hence $K(X, Y)=L(X, Y)$.

THEOREM 10. If $l_{1}$ is isomorphic to a subspace of $X$, then $K(X, X)$ is not a semi M-ideal.

Proof. Assume for contradiction that $l_{1}$ is isomorphic to a subspace of $X$ and that $K(X, X)$ is a semi $\mathrm{M}$-ideal. Let $\varepsilon>0$. Then there exists a linear operator $T: l_{1} \rightarrow X$ such that for all $x \in l_{1}$

$$
\|x\| \leqq\|T x\| \leqq\|x\|(1+\varepsilon)
$$


[15; Proposition 2.e.3]. In order to avoid technical complications, we will assume $\|x\|=\|T x\|$ for all $x \in l_{1}$. Let $Y=T\left(l_{1}\right)$. We will identify $Y$ with $l_{1}$. Let $e=(1,1, \ldots) \in \hat{\partial}_{e} Y_{1}^{*}$ and let $\tilde{e}$ be a normpreserving extension of $e$ to $X$. Let $x_{0}=(1,0, \ldots) \in \partial_{e} Y_{1} \cong X_{1}$. Define $S \in K(X, X)$ by

$$
S(x)=\tilde{e}(x) x_{0} .
$$

Then $S^{*}(f)=f\left(x_{0}\right) \tilde{e}$. For each $g \in \hat{\partial}_{e} Y_{1}^{*}$, let $\tilde{g}$ be a norm-preserving extension to $X$.

Suppose $U \in K(X, X)$ is such that

$$
U \in B(I+S, 1+\varepsilon) \cap B(I-S, 1+\varepsilon) .
$$

Then $\|S \pm(I-U)\| \leqq 1+\varepsilon$, and if $g \in \hat{\partial}_{e} Y_{1}^{*}$, then

$$
\begin{aligned}
1+\varepsilon & \geqq \max \left\|S^{*}(\tilde{\mathrm{g}}) \pm\left(\tilde{\mathrm{g}}-U^{*} \tilde{g}\right)\right\| \\
& =\max \left\|\tilde{\mathrm{g}}\left(x_{0}\right) \tilde{e} \pm\left(\tilde{\mathrm{g}}-U^{*} \tilde{\mathrm{g}}\right)\right\| \\
& =\max \left\|\tilde{e} \pm\left(\tilde{\mathrm{g}}-U^{*} \tilde{\mathrm{g}}\right)\right\| \\
& \geqq \max \left\|e \pm\left(g-\left.U^{*} \tilde{\mathrm{g}}\right|_{Y}\right)\right\| \\
& =1+\left\|g-\left.U^{*} \tilde{\mathrm{g}}\right|_{Y}\right\| .
\end{aligned}
$$

Therefore

$$
\left\|g-\left.U^{*} \tilde{g}\right|_{Y}\right\| \leqq \varepsilon
$$

But then if $g_{1}, g_{2} \in \hat{o}_{e} Y_{1}^{*}$ and $g_{1} \neq g_{2}$, then

$$
\begin{aligned}
& \left\|U^{*} \tilde{g}_{1}-U^{*} \tilde{g}_{2}\right\| \\
& \geqq\left\|\left.U^{*} \tilde{g}_{1}\right|_{Y}-\left.U^{*} \tilde{g}_{2}\right|_{Y}\right\| \\
& \geqq\left\|g_{1}-g_{2}\right\|-\left\|g_{1}-\left.U^{*} \tilde{g}_{1}\right|_{Y}\right\|-\left\|g_{2}-\left.U^{*} \tilde{g}_{2}\right|_{Y}\right\| \\
& \geqq 2-2 \varepsilon .
\end{aligned}
$$

If now $\varepsilon<\frac{1}{2}$, then this clearly contradicts that $U^{*}$ is compact.

We conclude with the following theorem.

THEOREM 11. Let $1<p<\infty$ and $p \neq 2$. Let $X=L_{p}(\mu)$ for some measure $\mu$. The following statements are equivalent:

1) $\mu$ is purely atomic.

2) $K(X, X)$ is an M-ideal in $L(X, X)$.

3) $K(X, X)$ is a semi M-ideal in $L(X, X)$. 
Proof. 1) $\Rightarrow$ 2) is proved in Theorem 4 .

2) $\Rightarrow 3$ ) is trivial.

$3) \Rightarrow 1)$. Assume $\mu$ is not purely atomic. We will show that $K(X, X)$ is not a semi M-ideal. Then it is well known that $L_{p}(0,1)$ is 1 -complemented in $L_{p}(\mu)$. Hence it follows from $\left({ }^{*}\right)$ that it is enough to show that $K(X, X)$ is not a semi M-ideal when $X=L_{p}(0,1)$. We can also assume $p>2$.

Let $\left(\chi_{n}\right)_{n=1}^{\infty}$ be the Haar basis in $L_{p}(0,1)$, and let $P_{n}\left(\sum_{k=1}^{\infty} a_{k} \chi_{k}\right)=\sum_{k=1}^{n} a_{k} \chi_{k}$ be the natural projections. $\left(\chi_{n}\right)_{n=1}^{\infty}$ is a monotone basis so $\left\|P_{n}\right\|=1$ for all $n$.

Let $1>\varepsilon>0$. Since $L_{p}(0,1)$ is uniformly convex, there exists $\varepsilon \geqq \delta>0$ such that if $\|x\| \leqq 1,\|y\| \leqq 1$ and $\|x-y\|>\varepsilon$, then $\|x+y\| \leqq 2(1-\delta)$ [8].

Assume for contradiction that $K(X, X)$ is a semi M-ideal. Then there exists $U \in K(X, X)$ such that

$$
\left\|I-U+P_{1}\right\|<1+\frac{\delta}{2}, \quad\left\|I-U-P_{1}\right\|<1+\frac{\delta}{2} .
$$

Since $\left\|\chi_{1}\right\|=1$, we get

$$
\left\|U \chi_{1}\right\|<1+\frac{\delta}{2}, \quad\left\|2 \chi_{1}-U \chi_{1}\right\|<1+\frac{\delta}{2} .
$$

If $2\left\|\chi_{1}-U \chi_{1}\right\| \geqq \varepsilon\left(1+\frac{1}{2} \delta\right)$, then by the uniform convexity, we get

$$
\left(1+\frac{\delta}{2}\right) 2(1-\delta) \geqq\left\|\left(2 \chi_{1}-U \chi_{1}\right)+U \chi_{1}\right\|=2 .
$$

This is a contradiction, hence

$$
\left\|\chi_{1}-U \chi_{1}\right\|<\frac{1}{2} \varepsilon\left(1+\frac{\delta}{2}\right)<\varepsilon .
$$

Hence we may assume $U \chi_{1}=\chi_{1}$ and

$$
\left\|I-U-P_{1}\right\|<1+2 \varepsilon .
$$

We define a sequence $\left(y_{k}\right)_{k=1}^{\infty}$ in $L_{p}(0,1)$ by

$$
y_{k}=\sum\left(\chi_{2^{k-1}+j}+\chi_{2^{k}+2 j-1}\right)
$$

where $j$ runs from 1 to $2^{k-1}$. Let $\mu$ be the Lebesgue measure on $(0,1)$. Then we have for all $k$

$$
\mu\left(\left\{x: y_{k}(x)=-1\right\}\right)=\frac{1}{2}
$$

and

$$
\mu\left(\left\{x: y_{k}(x)=2\right\}\right)=\frac{1}{4}=\mu\left(\left\{x: y_{k}(x)=0\right\}\right) .
$$


Define $K>0$ by $K^{p}=\frac{1}{4} 2^{p}+\frac{1}{2} 3^{p}$. We have $\left\|-2 \chi_{1}+y_{k}\right\|=K$ for all $k$. Since $p>2$, we have $\frac{1}{2} 4^{p}+1>3^{p}$. Hence there exists $c>0$ such that for all $k$

$$
\begin{aligned}
\left\|2 \chi_{1}+y_{k}\right\| & =\left(\frac{1}{4} 4^{p}+\frac{1}{4} 2^{p}+\frac{1}{2}\right)^{1 / p}=K+c . \\
& (1+2 \varepsilon)\left\|-2 \chi_{1}+y_{k}\right\| \\
\geqq & \left\|\left(I-U-P_{1}\right)\left(-2 \chi_{1}+y_{k}\right)\right\| \\
\geqq & \left\|2 \chi_{1}+y_{k}\right\|-\left\|U y_{k}\right\| \\
= & c+\left\|-2 \chi_{1}+y_{k}\right\|-\left\|U y_{k}\right\|
\end{aligned}
$$

such that

$$
c \leqq\left\|U y_{k}\right\|+2 \varepsilon K \text {. }
$$

Choosing $\varepsilon$ small enough, we get $\left\|U y_{k}\right\| \geqq \frac{1}{2} c$. This is impossible since $U$ is compact, so $K(X, X)$ can not be a semi M-summand.

\section{REFERENCES}

1. E. Alfsen and E. Effros, Structure in real Banach spaces, Ann. of Math. 96 (1972), 98-173.

2. J. Hennefeld, $A$ decomposition of $B(X)^{*}$ and unique Hahn-Banach extensions, Pacific J. Math. 46 (1973), 197-199.

3. E. H. Lacey, The isometric theory of classical Banach spaces, Die Grundlehren der math. Wissenschaften, Band 208, Springer-Verlag, Berlin - Heidelberg - New York, 1974.

4. A. J. Lazar, Polyhedral Banach spaces and extensions of compact operators, Israel J. Math. 7 (1969), 357-364.

5. A. Lima, Intersection properties of balls and subspaces in Banach spaces, Trans. Amer. Math. Soc. 227 (1977), 1-62.

6. Å. Lima, Intersection properties of balls in spaces of compact operators, Ann. Inst. Fourier (Grenoble) 28 (1978), 35-65.

7. J. Lindenstrauss, Extensions of compact operators, Mem. Amer. Math. Soc. 48 (1964).

8. J. Lindenstrauss and L. Tzafriri, Classical Banach spaces, Lecture Notes in Math., Vol. 338, Springer-Verlag, Berlin - Heidelberg - New York, 1973.

9. J. Mach and J. D. Ward, Approximation by compact operators in certain Banach spaces, J. Approximation Theory 23 (1978), 274-286.

10. N. J. Nielsen and G. H. Olsen, Complex preduals of $L_{1}$ and subspaces of $l_{\infty}^{n}(C)$, Math. Scand. 40 (1977), 271-287.

11. K. Saatkamp, M-ideals of compact operators, Math. Z. 158 (1978), 253-263.

12. R. R. Smith and J. D. Ward, M-ideal structure in Banach algebras, J. Functional Analysis 27 (1978), 337-349.

13. M. Zippin, On some subspaces of Banach spaces whose duals are $L_{1}$-spaces, Proc. Amer. Math. Soc. 23 (1969), 378-385.

14. J. Diestel and J. J. Uhl, Vector measures, American Mathematical Surveys, No. 15, 1977.

15. J. Lindenstrauss and L. Tzafriri, Classical Banach Spaces I, Sequence Spaces, Ergebnisse Math. Grenzgebiete, Bd. 92, Springer-Verlag, Berlin - Heidelberg - New York, 1977.

INSTITUTT FOR MATEMATISKE FAG

NORGES LANDBRUKSHØGSKOLE

1432 AS - NLH

NORGE 